\title{
Research progress on SLC7A11 in the regulation of cystine/cysteine metabolism in tumors (Review)
}

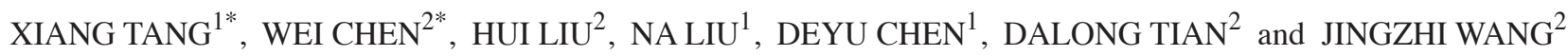 \\ ${ }^{1}$ Institute of Oncology, Affiliated Hospital of Jiangsu University, Zhenjiang, Jiangsu 212001; \\ ${ }^{2}$ Department of Radiotherapy Oncology, The Affiliated Yancheng First Hospital of Nanjing University Medical School, \\ The First People's Hospital of Yancheng, Yancheng, Jiangsu 224000, P.R. China
}

Received September 2, 2021; Accepted November 25, 2021

DOI: $10.3892 / \mathrm{ol} .2021 .13165$

\begin{abstract}
Solute carrier family 7 member 11 (SLC7A11) is a major transporter regulating cysteine metabolism and is widely expressed in a variety of tumor cells. SLC7A11 plays an important role in the occurrence, development, invasion and metastasis of tumors by regulating the transport of cysteine in the tumor microenvironment. SLC7A11 is expected to become a new therapeutic target and prognostic indicator for the individualized treatment of patients. According to relevant research reports, SLC7A11 can predict the stages and metastasis of liver, breast and lung cancer. Therefore, an in-depth exploration of the role of SLC7A11 in tumors may be important for the screening, early diagnosis, treatment and prognosis of patients with tumors. The current review summarizes the research progress on SLC7A11 in liver cancer, lung cancer and other tumors on the basis of previous primary studies. In addition, the present review systematically elaborates on the three main aspects of SLC7A11 pathways in some tumors, the cancer-promoting mechanisms, and the therapeutic relationship between SLC7A11 and tumors. Finally, the present review aims to provide a reference for assessing whether SLC7A11 can be used as a prognostic indicator and treatment target for tumor patients, and the future research direction with regard to SLC7A11 in tumors.
\end{abstract}

Correspondence to: Mr. Jingzhi Wang, Department of Radiotherapy Oncology, The Affiliated Yancheng First Hospital of Nanjing University Medical School, The First People's Hospital of Yancheng, 166 Yulong Road, Yancheng, Jiangsu 224000, P.R. China E-mail: 841179770@qq.com

Dr Deyu Chen, Institute of Oncology, Affiliated Hospital of Jiangsu University, 438 Jiefang Road, Zhenjiang, Jiangsu 212001, P.R. China E-mail: cdeyu@hotmail.com

*Contributed equally

Key words: solute carrier family 7 member 11, tumor, cysteine, ferroptosis

\section{Contents}

1. Introduction

2. Structure and biological function of cysteine

3. Structure and function of SLC7A11 and its regulatory mechanism

4. SLC7A11 regulates the association between cysteine metabolism and tumors

5. Future prospects

\section{Introduction}

With the continuous deepening and improvement of research on the tumor microenvironment and metabolic stress mechanisms, increasing evidence has shown that tumor cells can change cysteine metabolism through solute carrier family 7 member 11 (SLC7A11) to promote the occurrence and development of the tumor. The growth and migration of tumor cells are highly dependent on the tumor microenvironment, which is different from the normal tissue environment $(1,2)$. Given the rapid and massive proliferation of tumor cells, a microenvironment of metabolic stress, such as hypoxia and nutritional stress, is often formed. In theory, this metabolic stress microenvironment is not conducive to the survival and growth of tumors, but tumors can overcome the stress environment that is not conducive to their growth and continue to maintain the ability to proliferate indefinitely. The metabolic reprogramming of tumors (including changes in glucose and amino acid uptake) plays an important role in this. The results of correlation studies indicated that the tumor microenvironment exhibits the characteristics of homocysteine/cysteine (3) and is involved in tumor metabolic reprogramming and resistance to ferroptosis (4). Ferroptosis is due to the abnormal activation of the mitochondrial oxidative phosphorylation pathway due to iron overload, which produces high levels of lipid peroxides [e.g., reactive oxygen species (ROS)] when ATP is produced. Ferroptosis can be regarded as cell death caused by the accumulation of lipid peroxides on the cell membrane; it shows evident iron dependence and does not belong to the category of apoptosis or necrosis (5). From the morphological point of view, ferroptosis is characterized by mitochondrial atrophy, increased membrane density and reduced mitochondrial 
cristae, but does not show the typical morphological characteristics of traditional apoptosis, such as cell swelling, cell contraction, cell rupture, apoptotic body formation, and cytoskeleton disintegration and necrosis. In addition, ferroptosis does not have the typical characteristics of autophagy, such as autophagy vacuoles. Ferroptosis is closely related to reduced glutathione (GSH) metabolism, and GSH is predominantly synthesized by cysteine (6). Cystine in the microenvironment of tumor cells is predominantly transported into cells through the cystine/glutamate antiporter system xc- to participate in the synthesis of GSH, which leads to the resistance of tumor cells to ferroptosis, and promotes the occurrence and development of tumors (4). The cystine/glutamate antiporter system xc- is a member of the amino acid transporter family and consists of two subunits, i.e., light (SLC7A11) and heavy (SLC3A2) chain subunits. SLC7A11 plays a major biological function as a transporter; it can transport glutamate out of the cell and cystine into the cell at a ratio of 1:1. SLC3A2, as a chaperone protein, is involved in maintaining the stability of SLC7A11 only $(7,8)$.

The present review focuses on the unique role of SLC7A11 in the resistance of tumors to ferroptosis, and in the occurrence, development and treatment of tumors with regard to the regulation of cystine/cysteine metabolism.

\section{Structure and biological function of cysteine}

Cysteine (2-amino-3-mercaptopropionic acid), an aliphatic sulfhydryl-containing polar $\alpha$-amino acid, is a conditional amino acid that is essential for the human body and can be converted into cystine. Cysteine plays an important role in protein synthesis, catalysis, transport, post-translational modification and redox maintenance $(9,10)$. The two main functions of cysteine in the human body are its involvement in the production of reduced GSH and protein synthesis. GSH is a tripeptide composed of three amino acids, namely, cysteine, glutamic acid and glycine, and is one of the most important cellular antioxidants in the human body. Cysteine is the rate-limiting precursor of GSH synthesis, and its participation in the biosynthesis of GSH comprises two steps (11). The first step is the rate-limiting reaction, which uses cysteine and glutamate to synthesize $\gamma$-glutamyl-cysteine ( $\gamma$-Glu-Cys) by glutamate cysteine ligase. The second step is the addition of glycine to the C-terminus of $\gamma$-Glu-Cys by GSH synthetase to produce glutathione. Once synthesized, GSH becomes a detoxification substance for lipid peroxides. For example, glutathione peroxidase 4 (GPX4), which uses GSH as a cofactor, detoxifies lipid peroxides into lipid alcohols $(12,13)$. At the same time, GSH is oxidized into oxidized (GSSG), which then consumes $\mathrm{H}+$ in NADPH through GSH reductase and is reduced back to GSH, enabling GSH recycling. In addition, cysteine is a precursor or cofactor of other biomolecules with antioxidant properties, such as taurine, hydrogen sulfide and aconitic acid (14). Intracellular cysteine can be transformed from methionine and serine through sulfur transfer. In addition, cysteine can be obtained by the degradation and recovery of GSH and other proteins (15). However, due to the infinite proliferation characteristics of tumor cells, a tumor microenvironment of metabolic stress and oxidative stress, such as hypoxia and nutrient deficiency, is often formed. As a result, in this poor nutritional tumor microenvironment, the cysteine, which is provided through traditional biosynthesis or protein catabolism, cannot meet the high demand of the cancer cells for antioxidative defense and metabolic stress. Therefore, the method of obtaining cystine from an exogenous pathway through a nutrient transporter and then reducing it to cysteine by GAPDH has become the main strategy for most tumor cells. SLC7A11 is highly specific to cystine and glutamate, and is the main protein transporter for tumor cells to transport cysteine $(16,17)$.

\section{Structure and function of SLC7A11 and its regulatory mechanism}

Structure and function of SLC7A11. The human SLC7A11 gene is located on chromosome 4, contains 14 exons and consists of 502 amino acids. SLC7A11 is composed of 12 highly hydrophobic channel transmembrane proteins, the $\mathrm{N}$ - and C-termini of which are located in the cytoplasm (Fig. 1). SLC7A11 is widely expressed in normal tissues, such as those of the brain and liver, and cells, such as macrophages (18). In the internal environment of the human body, SLC7A11 can transport glutamate from the cell. At the same time, SLC7A11 can transport extracellular cystine into the cell. The cystine that enters the cytoplasm can be quickly reduced to cysteine by GAPDH. The acid is used for the synthesis of GSH. GSH, as a cofactor of the GPX family (such as GPX4), detoxifies lipid peroxide into lipid alcohol to protect tumor cells from oxidative stress, block the ferroptosis of tumor cells and promote tumor cell proliferation (Fig. 1). In addition, SLAC7A11 can regulate ferroptosis and participate in tumor proliferation and survival by regulating the nutritional dependence of glutamine and glucose (5).

Regulation mechanism of SLC7A11. The expression and activity of SLC7A11 are regulated by a variety of mechanisms, including transcription factors and epigenetic regulation.

Transcriptional regulation of SLC7A11 by transcription factors. Koppula et al (19) reported that under various stress conditions, such as oxidative stress and amino acid deficiency, the expression of SLC7A11 can be significantly upregulated, which is beneficial for cells to restore redox homeostasis and continue to survive under stress conditions. Activating transcription factor 4 (ATF4) and nuclear erythroid 2-related factor 2 (NRF2) are the two main transcription factors involved in the stress-induced transcription of SLC7A11. ATF4 is a member of the ATF/cAMP responsive element binding protein (ATF/CREB) transcription factor family and regulates redox homeostasis, amino acid metabolism and endoplasmic reticulum stress (20). When the living environment of a cell lacks amino acids, especially when glutamine and cysteine are lacking, the cell can transfer ATF4 into the nucleus through the general control nonderepressible 2 (GCN2)/eukaryotic initiation factor $2 \alpha(\mathrm{eIF} 2 \alpha) / \mathrm{ATF} 4$ signaling axis and bind to the amino acid response element (AARE) in the gene promoter, thereby promoting the transcription of genes involved in amino acid metabolism and stress response, including the transcription of SLC7A11 (21) (Fig. 2). NRF2 is a transcription factor that predominantly mediates the antioxidant response. Under normal physiological 


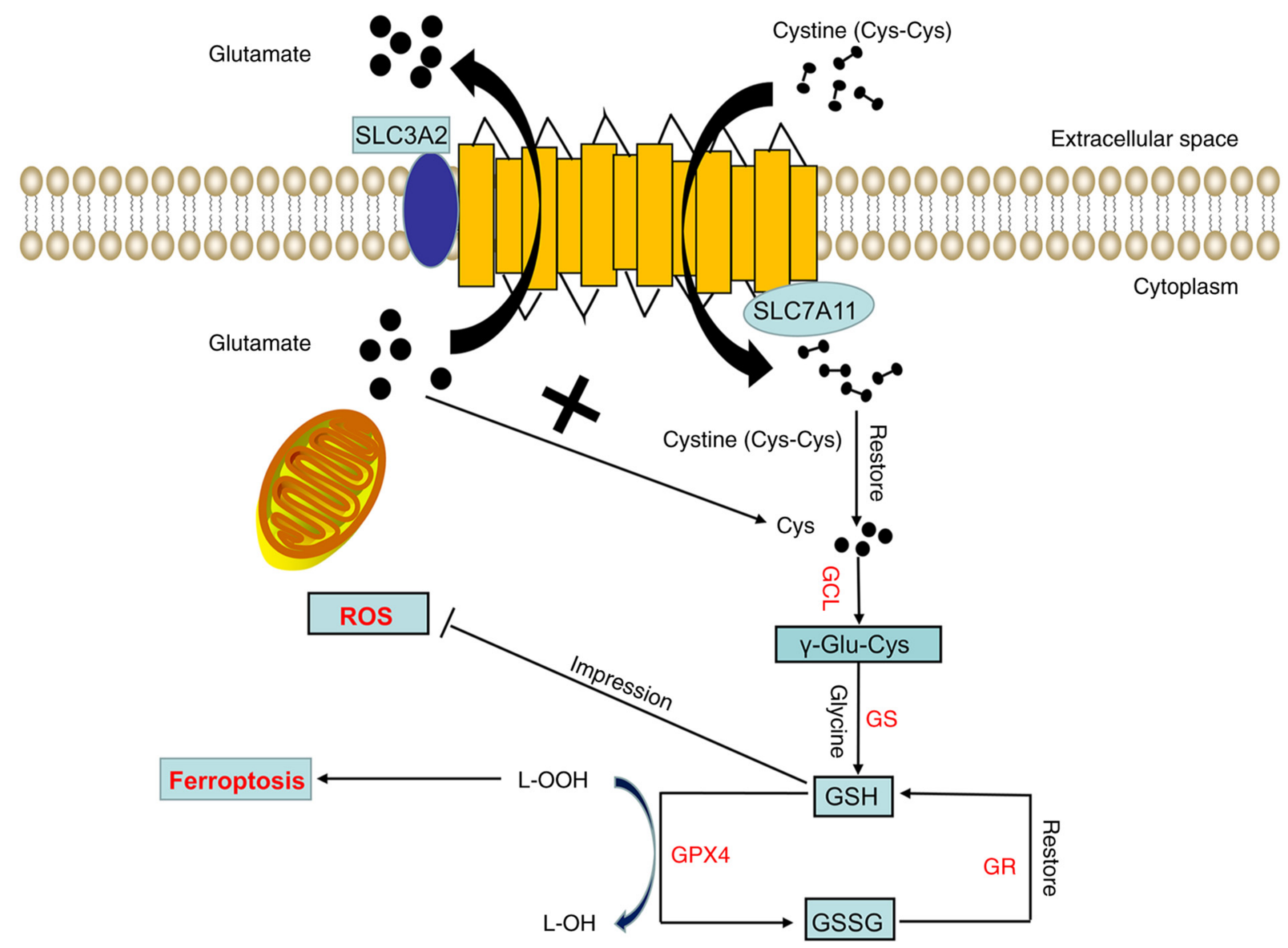

Figure 1. Structure and function of Xc-. The Xc- system consists of two subunits, namely, light (SLC7A11) and heavy (SLC3A2) chain subunits. SLC7A11 exerts the main biological function and transport activity, whereas SLC3A2 only participates in maintaining the stability of SLC7A11 as a chaperone protein. SLC7A11 transfers a molecule of extracellular cystine into the cell while transferring a molecule of intracellular glutamate out of the cell. The cystine transported into the cell is quickly reduced to cysteine. Next, cysteine and glutamic acid are combined under the action of GCL to form $\gamma$-glutamyl cysteine, and glycine is added to the C-terminus of $\gamma$-Glu-Cys through GS to produce reduced GSH. GPX4 then reduces lipid hydroperoxides into lipid alcohols through GSH, thereby inhibiting ferroptosis. At this time, GSH is oxidized into GSSG, which can be reduced to GSH again through GR. GCL, glutamate cysteine ligase; GS, glutathione synthetase; GPX4, glutathione peroxidase 4; GR, glutathione reductase; GSH, reduced glutathione; GSSG, oxidized glutathione; L-OOH, lipid hydroperoxide; L-OH, lipid alcohol; ROS, reactive oxygen species; SLC7A11, solute carrier family 7 member 11; SLC3A2, solute carrier family 3 member 2.

conditions, NRF2 is extremely unstable, can be ubiquitinated by Kelch-like ECH-associated protein 1 (KEAP1), and is rapidly degraded by the proteasome pathway. In the case of oxidative stress, the NRF2 degradation pathway mediated by KEAP1 is blocked, and NRF2 can remain stable and bind to the AARES region of the antioxidant response element to induce the transcription of a variety of antioxidant defense genes, including SLC7A11 (Fig. 2). ATF4 and NRF2 have been found to have a synergistic effect. Ye et al (22) reported that ATF4 and NRF2 can interact on the SLC7A11 promoter, and can synergistically regulate the SLC7A11 transcription under a variety of metabolic stress conditions.

Transcription factors can negatively regulate SLC7A11, and the main transcription factors for this are p53 and ATF3 (Fig. 2). The p53 protein is a transcription factor, and the gene is a tumor suppressor. A study has shown that p53 can promote ferroptosis in cells under various ferroptosis-inducing conditions. Part of its effect is achieved by inhibiting the expression of SLC7A11. p53 deficiency can evidently lead to the upregulation of SLC7A11, thereby enhancing the resistance of tumor cells to ferroptosis and inhibiting ferroptosis (23). One study has further revealed that p53 can inhibit the expression of
SLC7A11 by weakening the function of NRF2. ATF3 is another member of the ATF/CREB transcription factor family. Under basic conditions, ATF3 can bind to the SLC7A11 promoter and inhibit the expression of SLC7A11. However, under stress conditions that can induce the expression of SLC7A11, ATF3 does not inhibit the expression and activity of SLC7A11. Part of the role of Erastin (an inhibitor of SLC7A11) is achieved by upregulating ATF3 and promoting the ferroptosis of cells (24).

Overall, various stress conditions can partially promote the transcription of SLC7A11 through ATF4 and/or NRF2, whereas p53 and ATF3 predominantly inhibit the expression of SLC7A11 under basal conditions. These transcription factors affect the downstream biological effects (including cysteine metabolism) mediated by SLC7A11 by regulating the expression and transcriptional activity of SLC7A11, leading to changes in the sensitivity of cells to ferroptosis. In addition, SLC7A11 mRNA is directly regulated by miR-139-5p, miR-27a and miR-375 (25-27) (Fig. 2).

Transcriptional regulation of SLC7A11 by epigenetic modification. The epigenetic regulation of gene transcription is predominantly achieved through related modifications of 


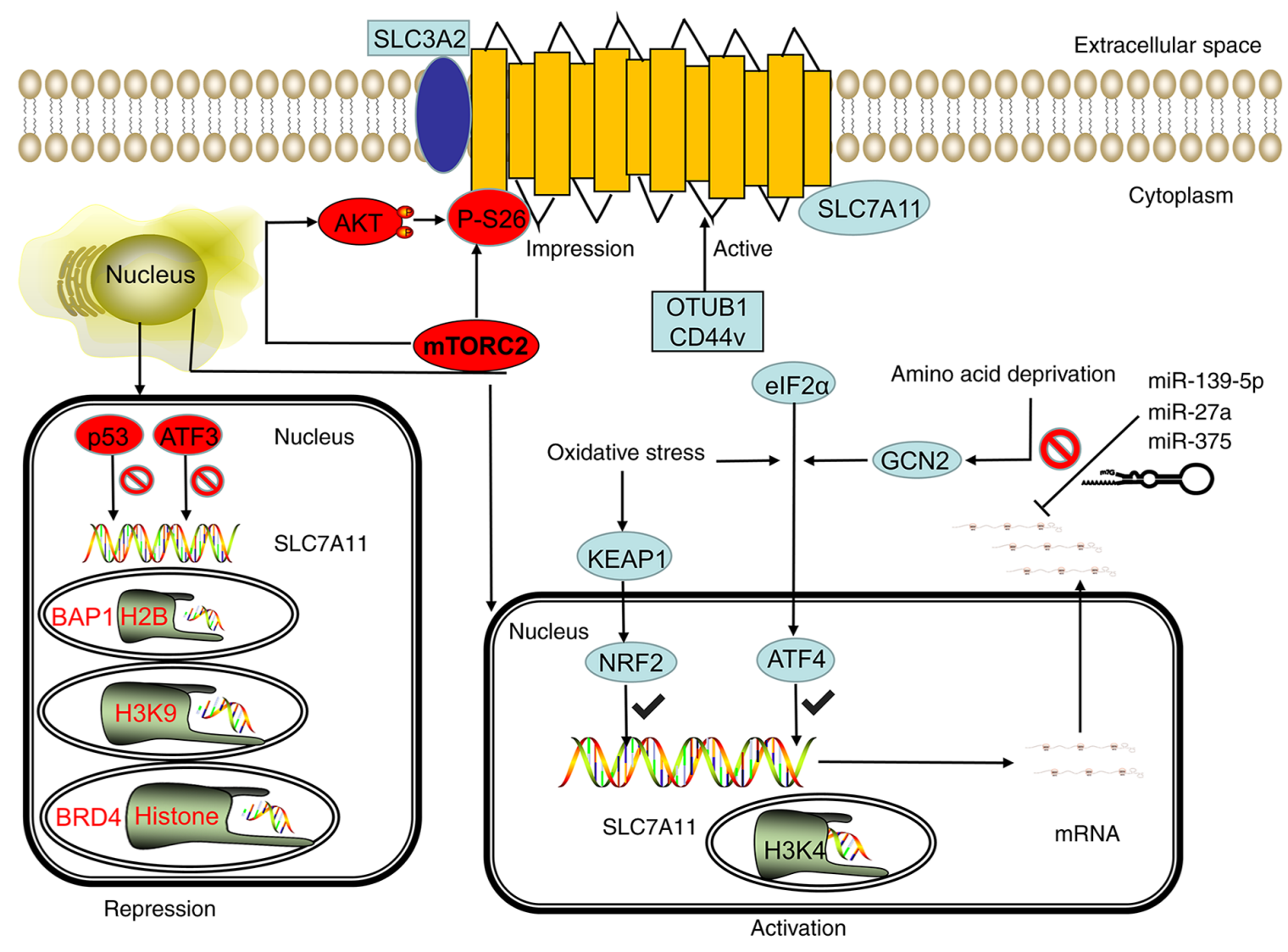

Figure 2. SLC7A11 is regulated by transcriptional, post-transcriptional and post-translational mechanisms. Under stress conditions, such as oxidative stress and amino acid deficiency, the GCN2-eIF2 $\alpha$ and KEAP1-NRF2 signal axes can induce SLC7A11 transcription, and the SWI/SNF chromatin remodeling complex can further promote the NRF2-mediated transcriptional activation of SLC7A11. However, p53 and ATF3 inhibit the expression of SLC7A11 under basal conditions. In epigenetics, the methylation of H3K4 can activate the transcription of SLC7A11, whereas the methylation of H3K9me3 and H3K27me3 can inhibit the transcription activity of SLC7A11. BRD4 can inhibit the transcription of SLC7A11 by regulating histone acetylation. The stability of SLC7A11 mRNA can be regulated by microRNA. miR-139-5p, mir-27a, and mir-375 can destroy the stability of SLC7A11 mRNA and inhibit its transcription. mTORC2 can regulate the phosphorylation of SLC7A11 at serine site 26 and inhibit the transport activity of SLC7A11 directly or through the AKT signaling pathway. CD44v and OTUB1 can inhibit the degradation of SLC7A11, thereby stabilizing the SLC7A11 protein and improving its transport activity. GCN2, general control nonderepressible 2; eIF2 $\alpha$, eukaryotic initiation factor 2 $\alpha$; KEAP1, kelch-like ECH-associated protein 1; NRF2, nuclear erythroid 2-related factor 2; mTORC2, mammalian target of rapamycin complex 2; OTUB1, OTU deubiquitinase ubiquitin aldehyde binding 1; ATF, activating transcription factor; p53, tumor protein 53; SWI/SNF, switch/sucrose non-fermentable modeling complex; BRD4, bromodomain-containing protein 4; miR, microRNA; CD44v, CD44 variant; SLC7A11, solute carrier family 7 member 11; SLC3A2, solute carrier family 3 member 2 .

DNA or DNA-related histones, such as DNA methylation, histone acetylation, methylation and ubiquitination. (28). A study showed that BRCA1-associated protein-1 (BAP1) is a nuclear protein with deubiquitinase that removes histone $\mathrm{H} 2 \mathrm{~A}$ monoubiquitylation (H2Aub) at position 119 of lysine. In a recent study, whole-genome analysis identified SLC7A11 as a key transcription target of BAP1 (29). At the SLC7A11 promoter, BAP1 deubiquitinates H2Aub and subsequently inhibits the expression of SLC7A11. The methylation of histone $\mathrm{H} 3$ (H3K9me3 and H3K27me3) at the lysine 9 and 27 positions also leads to the transcriptional inhibition of SLC7A11. Bromodomain-containing protein 4 (BRD4) is a member of the bromodomain and extra-terminal domain protein family. The main function of BRD4 is to recognize acetylated histones and recruit transcription factors to regulate gene transcription. Recent research showed that knocking out the BRD4 gene or using BRD4 inhibitors significantly inhibited the expression of SLC7A11 and promoted ferroptosis (Fig. 2) (30), indicating that BRD4 is likely to activate SLC7A11 transcription through epigenetic mechanisms. This phenomenon inhibits cell ferroptosis.
Chromatin remodeling is another key epigenetic mechanism that controls genes. Recently, studies showed that switch/sucrose non-fermentable (SWI/SNF) complexmediated chromatin remodeling is involved in regulating SLC7A11 transcription. The SWI/SNF complex combines with the SLC7A11 promoter to promote the NRF2-mediated transcriptional activation of SLC7A11 through the chromatin remodeling of the SWI/SNF complex. SWI/SNF deficiency leads to the suppression of SLC7A11 transcription, which causes impaired cystine uptake and GSH biosynthesis, and subsequently promotes ROS-induced cell ferroptosis (31).

It was recently found that the adhesion molecule CD44 variant $(\mathrm{CD} 44 \mathrm{v})$ forms a complex by binding to SLC7A11, thereby maintaining the stability of SLC7A11. The inactivation of CD44v destroys the stability of SLC7A11 and promotes ferroptosis (32). In addition, mammalian target of rapamycin complex 2 can directly phosphorylate SLC7A11 at serine 26 and serine 25 through the AKT signaling pathway $(33,34)$ (Fig. 2).

In summary, these studies confirmed that SLC7A11 can be regulated by a variety of post-translational mechanisms, 
including regulation of protein stability, localization and transporter activity.

\section{SLC7A11 regulates the association between cysteine metabolism and tumors}

Cysteine is involved in the synthesis of GSH, and GSH can be used as a cofactor of GPX. Lipid peroxides detoxify into lipid alcohol, thereby protecting tumor cells from oxidative stress and blocking the ferroptosis of tumor cells. Tumor cells obtain cysteine from outside the cell through SLC7A11 to maintain the level of homo-GSH in the cell, thereby meeting the high energy demand against oxidative stress. This phenomenon is conducive to the survival and development of tumor cells. SLC7A11 can also participate in the metabolic reprogramming of tumors by regulating cysteine metabolism, and promote tumor growth and migration, such as via increasing ingestion of glutamine (35).

Next, the present review specifically discusses the association between the regulation of cysteine metabolism and tumor occurrence, development and prognosis by SLC7A11. The study attempts to show that SLC7A11 is widely expressed in various tumors of the human body and can be a potential therapeutic target for a number of systems.

SLC7A11 regulates cysteine metabolism and liver cancer. The etiology of liver cancer is related to environment, diet or lifestyle factors (36). Evidence shows that liver cancer is closely related to hepatitis $\mathrm{B} / \mathrm{C}$ virus infection and liver cirrhosis (37). There are 700,000 new liver cancer patients worldwide each year, and cases in China account for more than half of the global total. Given the low sensitivity of liver cancer to radiotherapy and chemotherapy, the current main treatments are surgery and liver transplantation. However, as liver cancer cells are prone to metastasis, these treatments often fail to achieve the expected results (38). Therefore, finding new and effective targets for the diagnosis and treatment of liver cancer has become an urgent problem to be solved. Kinoshita et al (39) showed the association between SLC7A11 in mRNA transcription and the clinical characteristics of hepatoceullar carcinoma, and proposed that SLC7A11 has prognostic value in this disease. However, the study also reported that the expression of SLC7A11 protein was detected in only one out of eight liver cancer specimens (39). Recent studies have found that compared with that in normal tissues and cells, SLC7A11 expression is high at the protein level in liver cancer tissues and cells, and is significantly related to an advanced poor prognosis (40), further confirming that SLC7A11 can be used as an independent prognostic factor of liver cancer factor. A further study by Wada et al (41) found that SLC7A11 is highly expressed in poorly differentiated liver cancer. In vitro experiments also confirmed that the SLC7A11Z inhibitor sulfasalazine (SASP) could inhibit the uptake of cysteine, reduce the resistance of liver cancer cells to ROS and lipid peroxide, and enhance the ferroptosis of liver cancer cells by inhibiting CD44v9-SLC7A11 (41).

The liver is the organ with the highest expression and activity of cysteine dioxygenase (CDO) protein in the human body. $\mathrm{CDO}$ occupies a key position in the normal synthesis and degradation of cysteine in the human body and is an important biological enzyme of cysteine, which is obtained through the diet (9). The development of liver cancer often follows the trilogy of hepatitis-cirrhosis-liver cancer. Abnormalities in liver function are present at each step, but whether this leads to abnormalities in CDO needs further investigation. We hypothesize that the occurrence and development of liver cancer is closely related to the abnormal physiological pathway of cysteine, and that iver cancer cells can obtain increased cysteine from extracellular pathways by upregulating the expression of SLC7A11 in the presence of toxic substances and oxidative stress. This aspect is a direction worth exploring in the future. In addition, SLC7A11 is closely related to the ferroptosis resistance and glutamine deprivation of liver cancer cells. Therefore, SLC7A11 could be a potential target for the treatment of liver cancer.

SLC7A11 regulates cysteine metabolism and lung cancer. The incidence and mortality of lung cancer rank first in the world, and the rates are increasing every year (42). With the aging of the Chinese population, the incidence of lung cancer is predicted to continue to rise. Traditional treatment options for lung cancer include surgery, radiation therapy and chemotherapy (43). In recent years, the targeted therapy of lung cancer has received increasing attention and recognition. Programmed cell death protein 1 (PD-1), PD ligand protein-1 and cytotoxicity $\mathrm{T}$ lymphocyte-associated antigen-4 inhibitors showed good effects (increased survival time and improve survival rate) in the treatment of small cell lung cancer (SCLC) and non-SCLC (NSCLC). However, as treatment progresses, most patients eventually develop resistance (44). Therefore, finding new targets for the treatment of lung cancer is important to improve the survival and quality of life of patients with this disease. Recent studies showed that SLC7A11 was overexpressed in lung adenocarcinoma, squamous cell carcinoma and NSCLC. The follow-up analysis of 254 patients with NSCLC found that patients with high expression of SLC7A11 tended to be at later stages and that the survival times were significantly shortened (45). These results confirmed that the expression of SLC7A11 is significantly correlated with cancer stage and 5-year overall survival rate. SLC7A11 decreases the GSH/GSSG ratio through overexpression, thereby creating an oxidized intracellular microenvironment in multiple lung cancer cell lines and promoting tumor growth. When the inhibitor Erastin is used to target the expression of SLC7A11, the growth and proliferation of lung cancer cells are significantly inhibited (45). In normal tracheal epithelial cells, SLC7A11 has been shown to participate in the metabolic reprogramming of the cells by regulating cystine metabolism, which is likely to be closely related to the occurrence of lung cancer (45). At present, the occurrence of lung cancer is known to be related to a variety of gene mutations, such as the translocation of EGFR, TP53, KRAS, ALK, RET or ROS1. Recent studies by Hu et al (46) confirmed that the KRAS mutant gene of lung adenocarcinoma acts on NRF2 through its downstream pathways (including MAPK, PI3K/AKT and RAS-related protein pathways), regulates the expression of SLC7A11, upregulates the levels of intracellular cysteine and GSH, and then promotes the progression of the tumor (Fig. 3). Compared with patients with lung cancer without KRAS mutation, those with lung cancer and KRAS mutations have significantly 


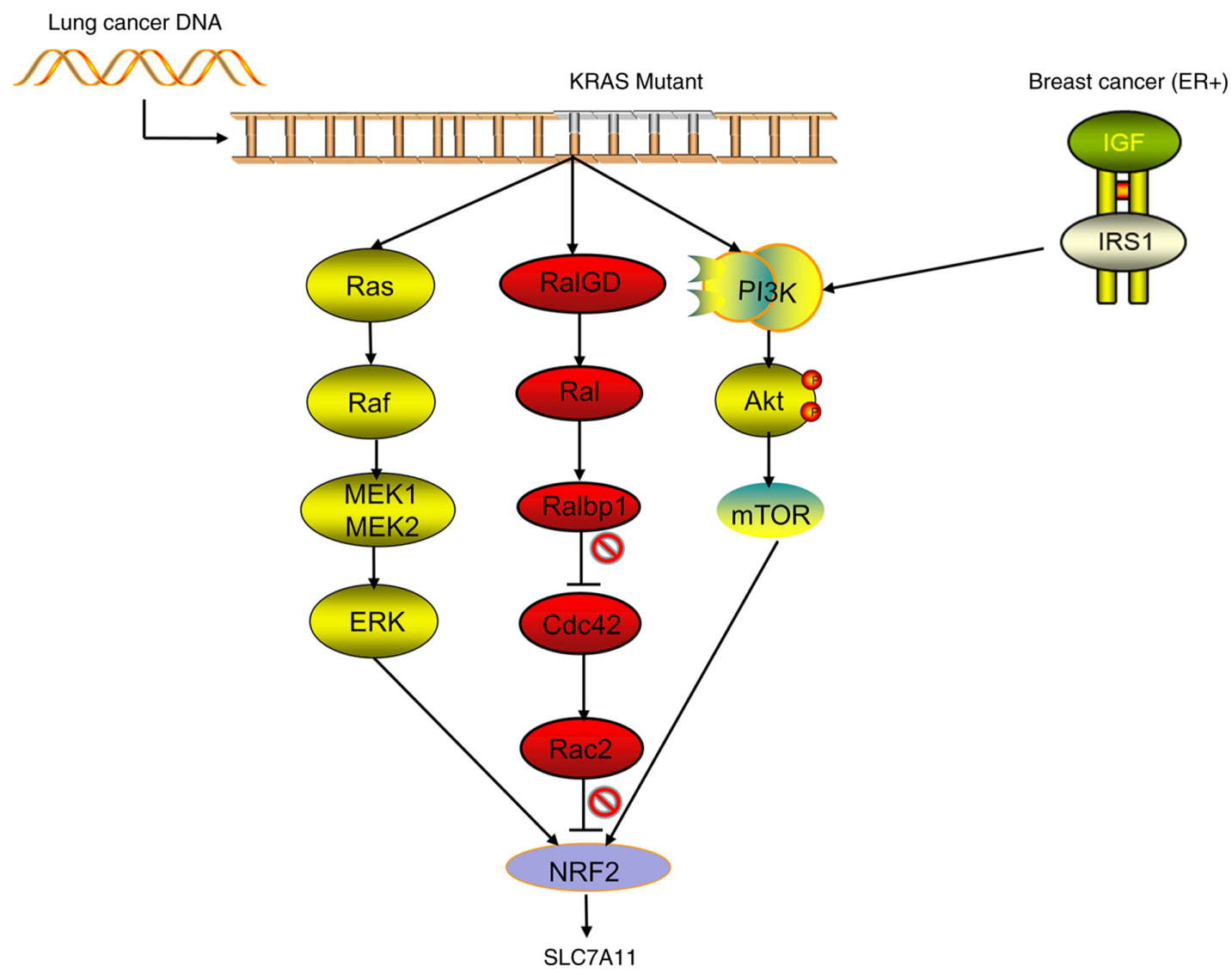

Figure 3. Signal pathways regulating the transcriptional activity of SLC7A11. Lung cancer in patients is often accompanied by mutations in the KRAS gene, and mutant KRAS acts on NRF2 by activating its downstream pathways (including the classic MAPK, PI3K/AKT and RAL pathways) to promote the transcriptional activity of SLC7A11. In addition, in patients with estrogen receptor-positive breast cancer, breast cancer cells can upregulate the transcriptional activity of SLC7A11 through the IGF-I/IRS-1/PI3K signaling axis. NRF2, nuclear erythroid 2-related factor 2; ER, estrogen receptor; SLC7A11, solute carrier family 7 member 11; RAL, RAS-related protein.

increased expression levels of NRF2 and SLC7A11, significantly upregulated intracellular cysteine and a significantly decreased overall survival rate (46). Wang et al (47) found that in NSCLC, the TP53 mutant has lost the functions of inducing cell cycle arrest and senescence, but still plays a certain role in inhibiting tumors by blocking the function of SLC7A11 (47). However, Jennis et al (48) constructed a TP53 mutant that retained the function of inducing cell cycle arrest, but could not inhibit the function of SLC7A11, and found that the tumor suppressor effect of the mutant was markedly decreased (48). Therefore, we hypothesize that the occurrence of NSCLC is closely related to TP53 and KRAS mutations, but that the pathogenesis is predominantly a series of functional abnormalities, such as TP53/KRAS-SLC7A11 cysteine ferroptosis rather than the abnormalities of cell cycle regulation caused by TP53 mutation in the traditional sense. Studies showed that CD44v and SLC7A11 are involved in the resistance of lung cancer to cisplatin and other drugs. The use of SASP can improve the drug resistance of lung cancer and promote the death of tumor-related stem cells $(49,50)$. The relationship among EGFR, ALK and RET or between ROS1 and SLC7A11 needs to be further explored. SPAP plays an important role in inhibiting tumor growth and promoting ferroptosis, but requires a large dose to inhibit SLC7A11, thereby limiting the clinical application of SPAP. Therefore, Hu et al (46) also screened out the compound HG106, which has achieved good effects in lung cancer modeling mice (46). These studies showed that SLC7A11 is involved in the occurrence and drug resistance of lung cancer and is closely related to the survival rate and prognosis of patients. Therefore, SLC7A11 can be used as a potential site for the molecular targeted therapy of lung cancer. At present, relevant research and clear evidence to support SLC7A11 driving the mutations of genes, such as TP53 and KRAS, and the specific mechanisms driving the aforementioned gene mutations remain lacking. This gap may be the key research direction of SLC7A11 in the field of lung cancer in the future.

SLC7A11 and breast cancer. Breast cancer is the most common malignant tumor in women and the main cause of death in women with cancer (42). The full use of antiestrogen hormone drugs (e.g., tamoxifen and aromatase inhibitors) and trastuzumab therapy (Herceptin) has benefited patients with breast cancer, but metastasis and relapse affect the survival of patients, especially in those with triple-negative breast cancer (TNBC) (51). Therefore, targeted and minimally toxic treatments for breast cancer are needed. SLC7A11 is reported to be highly expressed in a variety of breast cancer cells. Breast cancer cells with high expression of SLC7A11 have strong resistance to oxidative stress and ferroptosis, but are sensitive to the nutritional deprivation of glucose (52). Along with the high expression of SLC7A11, glucose deprivation 
can increase the ROS level of breast cancer cells through the AMPK/ACC pathway, thereby promoting the death of breast cancer cells (52). In estrogen receptor-positive breast cancer, the expression of SLC7A11 can be upregulated through the IGF-1/IRS-1/PI3K signaling axis to improve the ability of the tumor to withstand oxidative stress, which is beneficial to the survival of tumor cells and the occurrence of chemotherapy resistance (53) (Fig. 3). Ruiu et al (54) showed that the use of anti-SLC7A11 antibody vaccines to inhibit the expression of SLC7A11 can increase the sensitivity of breast cancer to chemotherapy (such as doxorubicin) and molecular targeted (such as PD-1) drugs. In addition, the use of this vaccine can induce autoimmune responses by targeting differentiated cancer stem cells, thereby achieving good therapeutic effects and having minimal toxicity. The expression can also be used to diagnose and treat the autoimmune response of tumor stem cells to achieve good results and have an excellent response, such as killing tumor cells, limiting tumor metastasis and exhibiting a less immunotoxic response (54). Conti et al (55) also confirmed that anti-HER2 vaccine predominantly inhibits tumor growth and that anti-SLC7A11 vaccine predominantly inhibits tumor metastasis, thereby showing that SLC7A11 can be used as a breast cancer treatment target. However, studies on the relationship between SLC7A11 and TNBC are few. Given the limited available treatments for TNBC, new methods are urgently needed. Therefore, the relationship between SLC7A11 and TNBC still needs to be further explored, and SLC7A11 still has marked prospects as a breast cancer treatment target.

SLC7A11 and glioma. Glioma is the most common malignant primary brain tumor. The rapid growth of this tumor benefits from the tumor-mediated release of glutamate, and the release of glutamate from gliomas is considered to cause the death of peritumoral neurons and make room for tumor growth (56). A previous study showed that the expression of SLC7A11 in the tumor tissues of $\sim 50 \%$ of patients with glioma was higher than that in normal tissues adjacent to the cancer. Compared with those lacking the transporter, tumors with high expression of SLC7A11 had strong proliferation ability and could produce increased excitotoxicity of glutamate, thus shortening the overall survival of the patients (57). This phenomenon indicates that SLC7A11 can be used as an independent prognostic indicator for patients with glioma. SLC7A11 is reported to promote the occurrence and development of gliomas by participating in the promotion of tumor-associated epilepsy, an important cause of glioma in patients. Although various brain tumors may induce seizures, the highest incidence and prevalence of epilepsy is found within patients with glioma $(\leq 80 \%)(58,59)$. Consistent with the research in lung cancer, Polewski et al (60) also found in glioma that SLC7A11 transports cysteine into tumor cells, participates in the synthesis of GSH and downregulates cellular ROS levels, thereby generating increased cancer-like stem cells. The functions of SLC7A11 and tumor stem cells are basically the same and are involved in the recurrence and metastasis of glioma. SLC7A11 may be regulated by EGFR/CD44 in glioma cells (61). The study by Long et al (62) showed that treatment with VEGF blockers can lead to increased expression of SLC7A11. In addition, the study pointed out that the glutamate output by SLC7A11 from tumor cells can promote the proliferation of regulatory $\mathrm{T}$ cells and improve the immunosuppressive function, thereby helping in improving the adaptability and drug resistance of glioblastoma against VEGF treatment (62). Robert et al (57) also found that the use of SLC7A11 inhibitors or targeted interference downregulates SLC7A11 levels due to cysteine uptake disorders, and that the resistance of the tumor to ROS is weakened, which can significantly increase the death of glioma cells (57). Although in vivo experiments need to be further improved, the aforementioned studies have confirmed that SLC7A11 is closely related to the prognosis, recurrence and metastasis of glioma, and that it is expected to become a new therapeutic target for glioma.

In addition, existing studies have also shown the relationship between SLC7A11 and pancreatic cancer, colorectal cancer and lymphoma in terms of prognosis and metastasis, and that SLC7A11 also plays a role in promoting cancer $(25,63,64)$, but the conclusions and mechanisms of action need further experimental verification.

\section{Future prospects}

SLC7A11, as an amino acid transport active subunit on the cell membrane, exerts a wide range of biological effects in organisms. At the same time, abnormality in the regulatory mechanism of SLC7A11 plays an important role in the process of tumor invasion, migration and drug resistance in multiple systems, such as the digestive, respiratory, reproductive and nervous systems. SLC7A11 is an important biomarker for tumor diagnosis and prognosis in various systems. Evidence has indicated that SLC7A11 can be used as a broad target for tumor treatment. At present, the two strategies with regard to SLC7A11 are the direct inhibition of SLC7A11-mediated cystine uptake and the targeting of SLC7A11-induced glucose or glutamine dependence. The first strategy occurs via the direct use of SLC7A11 inhibitors, such as SASP, to inhibit its uptake of cystine, antagonize its role in tumor resistance and tumor stem cell formation, and improve tumor treatment efficacy. At present, these studies have achieved only preliminary results. A number of clinical trials on SASP in tumor resistance and stem cell therapy (e.g., EPOC1205, EPOC1407 and UMIN00017854) are underway. In addition, a vaccine against SLC7A11 has been created, but the safety of the vaccine needs to be further explored, as it may require a large dose to achieve the inhibitory effect, and any toxicity and side effects should be assessed. Therefore, the development of new high-efficiency small-molecule drugs targeting SLC7A11 is one of the development directions of tumor treatment. The second strategy is to select a glucose transporter or glutaminase inhibitors for the treatment of SLC7A11 overexpression tumors, which can induce tumor cell death by using the high sensitivity of SLC7A11 overexpression tumors to glucose and glutamine deficiency. Recent studies showed that the combination of immunotherapy (or radiotherapy) and SLC7A11 inhibitors (e.g., imidazole ketone erastin sulfadiazine and sorafenib) has a strong therapeutic effect $(65,66)$. In addition, SLC7A11 promotes the synthesis of GSH by transporting cysteine, thereby increasing the drug resistance of various tumors, such as lung cancer, breast cancer and glioma. The drug resistance caused by SLC7A11 is also related to the activation of MAPK and other pathways. The development of 
drugs that inhibit or block these pathways can reverse tumor resistance and enhance the efficacy of tumor radiotherapy and chemotherapy. After overcoming various limitations and drawbacks, SLC7A11 may become a new prognostic indicator and a new therapeutic target for tumors in the future.

\section{Acknowledgements}

Not applicable.

\section{Funding}

Not applicable.

\section{Availability of data and materials}

Not applicable.

\section{Authors' contributions}

XT and JW searched the literature and drafted the manuscript. WC and HL performed revisions to the manuscript. DC reviewed and edited the manuscript. NL and DT were involved in the conception of the study. All authors read and approved the final manuscript. Data authentication is not applicable.

\section{Ethics approval and consent to participate}

Not applicable.

\section{Patient consent for publication}

Not applicable.

\section{Competing interests}

The authors declare that they have no competing interests.

\section{References}

1. Zheng $\mathrm{J}$ and Gao P: Toward normalization of the tumor microenvironment for cancer therapy. Integr Cancer Ther 18: 1534735419862352,2019

2. Bissell MJ and Hines WC: Why don't we get more cancer? A proposed role of the microenvironment in restraining cancer progression. Nat Med 17: 320-329, 2011.

3. Meng X, Yang Y, Zhou L, Zhang L, Lv Y, Li S, Wu Y, Zheng M, Li W, Gao G, et al: Dual-responsive molecular probe for tumor targeted imaging and photodynamic therapy. Theranostics 7 : 1781-1794, 2017.

4. Stockwell BR, Angeli JP, Bayir H, Bush AI, Conrad M, Dixon SJ, Fulda S, Gascón S, Hatzios SK, Kagan VE, et al: Ferroptosis: A regulated cell death nexus linking metabolism, redox biology, and disease. Cell 171: 273-285, 2017.

5. Dixon SJ, Lemberg KM, Lamprecht MR, Skouta R, Zaitsev EM, Gleason CE, Patel DN, Bauer AJ, Cantley AM, Yang WS, et al: Ferroptosis: An iron-dependent form of nonapoptotic cell death. Cell 149: 1060-1072, 2012.

6. Dolma S, Lessnick SL, Hahn WC and Stockwell BR: Identification of genotype-selective antitumor agents using synthetic lethal chemical screening in engineered human tumor cells. Cancer Cell 3: 285-296, 2003

7. Sato H, Tamba M, Ishii T and Bannai S: Cloning and expression of a plasma membrane cystine/glutamate exchange transporter composed of two distinct proteins. J Biol Chem 274: 11455-11458, 1999.
8. Kandasamy P, Gyimesi G, Kanai Y and Hediger MA: Amino acid transporters revisited: New views in health and disease. Trends Biochemical Sci 43: 752-789, 2018.

9. Stipanuk MH, Dominy JE Jr, Lee JI and Coloso RM: Mammalian cysteine metabolism: New insights into regulation of cysteine metabolism. J Nutr 136 (Suppl 6): 1652S-1659S, 2006.

10. Combs JA and DeNicola GM: The non-essential amino acid cysteine becomes essential for tumor proliferation and survival. Cancers (Basel) 11: 678, 2019.

11. Lu SC: Regulation of glutathione synthesis. Mol Aspects Med 30: 42-59, 2009.

12. Friedmann JP, Schneider M, Proneth B, Tyurina YY, Tyurin VA, Hammond VJ, Herbach N, Aichler M, Walch A, Eggenhofer E, et al: Inactivation of the ferroptosis regulator Gpx4 triggers acute renal failure in mice. Nat Cell Biol 16: 1180-1191, 2014.

13. Yang WS, SriRamaratnam R, Welsch ME, Shimada K, Skouta R, Viswanathan VS, Cheah JH, Clemons PA, Shamji AF, Clish CB, et al: Regulation of ferroptotic cancer cell death by GPX4. Cell 156: 317-331, 2014.

14. Scalise M, Pochini L, Pingitore P, Hedfalk K and Indiveri C: Cysteine is not a substrate but a specifific modulator of human ASCT2 (SLC1A5) transporter. FEBS Lett 589: 3617-3623, 2015.

15. Hanigan MH and Ricketts WA: Extracellular glutathione is a source of cysteine for cells that express gamma-glutamyl transpeptidase. Biochemistry 32: 6302-6306, 1993.

16. Zerbib E, Arif T, Shteinfer-Kuzmine A, Chalifa-Caspi V and Shoshan-Barmatz V: VDAC1 silencing in cancer cells leads to metabolic reprogramming that modulates tumor microenvironment. Cancers (Basel) 13:2850, 2021.

17. Lei G, Zhang Y, Koppula P, Liu X, Zhang J, Lin SH, Ajani JA, Xiao Q, Liao Z, Wang $\mathrm{H}$, et al: The role of ferroptosis in ionizing radiation-induced cell death and tumor suppression. Cell Res 30: 146-162, 2020.

18. Fotiadis D, Kanai Y and Palacín M: The SLC3 and SLC7 families of amino acid transporters. Mol Aspects Med 34: 139-158, 2013.

19. Koppula P,Zhang Y, Shi J, Li W and Gan B: The glutamate/cystine antiporter SLC7A11/xCT enhances cancer cell dependency on glucose by exporting glutamate. J Biol Chem 292: 14240-14249, 2017.

20. Pakos-Zebrucka K, Koryga I, Mnich K, Ljujic M, Samali A and Gorman AM: The integrated stress response. EMBO Rep 17: 1374-1395, 2016.

21. Kilberg MS, Shan J and Su N: ATF4-dependent transcription mediates signaling of amino acid limitation. Trends Endocrinol Metab 20: 436-443, 2009.

22. Ye P, Mimura J, Okada T, Sato H, Liu T, Maruyama A, Ohyama C and Itoh K: Nrf2-and ATF4-dependent upregulation of xCT modulates the sensitivity of T24 bladder carcinoma cells to proteasome inhibition. Mol Cell Biol 34: 3421-3434, 2014.

23. Jiang L, Kon N, Li T, Wang SJ, Su T, Hibshoosh H, Baer R and $\mathrm{Gu}$ W: Ferroptosis as a p53-mediated activity during tumour suppression. Nature 520: 57-62, 2015.

24. Clemons NJ, Liu DS, Duong CP and Phillips WA: Inhibiting system $\mathrm{xC}(-)$ and glutathione biosynthesis-a potential Achilles'hell in mutant-p53. Mol Cell Oncol 4: e1344757, 2017.

25. Zhu JH, De Mello RA, Yan QL, Wang JW, Chen Y, Ye QH, Wang ZJ, Tang HJ and Huang T: MiR-139-5p/SLC7A11 inhibits the proliferation, invasion and metastasis of pancreatic carcinoma via PI3K/Akt signaling pathway. Biochim Biophys Acta Mol Basis Dis 1866: 165747, 2020.

26. Drayton RM, Dudziec E, Peter S, Bertz S, Hartmann A, Bryant HE and Catto JW: Reduced expression of miRNA-27a modulates cisplatin resistance in bladder cancer by targeting the cystine/glutamate exchanger SLC7A11. Clin Cancer Res 20: 1990-2000, 2014.

27. Wu Y, Sun X, Song B, Qiu X and Zhao J: MiR-375/SLC7A11 axis regulates oral squamous cell carcinoma proliferation and invasion. Cancer Med 6: 1686-1697, 2017.

28. Jaenisch R and Bird A: Epigenetic regulation of gene expression: How the genome integrates intrinsic and environmental signals. Nat Genet 33 (Suppl): S245-S254, 2003.

29. Zhang Y, Shi J, Liu X, Feng L, Gong Z, Koppula P, Sirohi K Li X, Wei Y, Lee H, et al: BAP1 links metabolic regulation of ferroptosis to tumour suppression. Nat Cell Biol 20: 1181-1192, 2018.

30. Sui S, Zhang J, Xu S, Wang Q, Wang P and Pang D: Ferritinophagy is required for the induction of ferroptosis by the bromodomain protein BRD4 inhibitor (+)-JQ1 in cancer cells. Cell Death Dis 10: 331, 2019. 
31. Ogiwara H, Takahashi K, Sasaki M, Kuroda T, Yoshida H, Watanabe R, Maruyama A, Makinoshima H, Chiwaki F, Sasaki $\mathrm{H}$, et al: Targeting the vulnerability of glutathione metabolism in ARID1A defificient cancers. Cancer Cell 35 177-190, 2019.

32. Liu T, Jiang L, Tavana $\mathrm{O}$ and Gu W: The deubiquitylase OTUB1 mediates ferroptosis via stabilization of SLC7A11. Cancer Res 79: 1913-1924, 2019.

33. Gu Y, Albuquerque CP, Braas D, Zhang W, Villa GR, Bi J, Ikegami S, Masui K, Gini B, Yang H, et al: mTORC2 regulates amino acid metabolism in cancer by phosphorylation of the cystineglutamate antiporter xCT. Mol Cell 67: 128-138, 2017.

34. Kim J and Guan KL: mTOR as a central hub of nutrient signalling and cell growth. Nat Cell Biol 21: 63-71, 2019.

35. Goji T, Takahara K, Negishi M and Katoh H: Cystine uptake through the cystine/glutamate antiporter xCT triggers glioblastoma cell death under glucose deprivation. J Biol Chem 292: 19721-19732, 2017.

36. Schlageter M, Terracciano LM, D'Angelo S and Sorrentino P Histopathology of hepatocellular carcinoma. World J Gastroenterol 20: 15955-15964, 2014.

37. Cabibbo $\mathrm{G}$, Maida $\mathrm{M}$, Genco $\mathrm{C}$, Antonucci $\mathrm{M}$ and Cammà $\mathrm{C}$ : Causes of and prevention strategies for hepatocellular carcinoma. Semin Oncol 39: 374-383, 2012.

38. Liu L, Zhu XD, Wang WQ, Shen Y, Qin Y, Ren ZG, Sun HC and Tang ZY: Activation of beta-catenin by hypoxia in hepatocellular carcinoma contributes to enhanced metastatic potential and poor prognosis. Clin Cancer Res 16: 2740-2750, 2010.

39. Kinoshita H, Okabe H, Beppu T, Chikamoto A, Hayashi H, Imai K, Mima K, Nakagawa S, Ishimoto T, Miyake K, et al: Cystine/glutamic acid transporter is a novel marker for predicting poorsurvival in patients with hepatocellular carcinoma. Oncol Rep 29: 685-689, 2013.

40. Kim DH, Kim WD, Kim SK, Moon DH and Lee SJ: TGF- 11 -mediated repression of SLC7A11 drives vulnerability to GPX4 inhibition in hepatocellular carcinoma cells. Cell Death Dis 11: 406, 2020

41. Wada F, Koga H, Akiba J, Niizeki T, Iwamoto H, Ikezono Y, Nakamura T, Abe M, Masuda A, Sakaue T, et al: High expression of CD44v9 and $\mathrm{xCT}$ in chemoresistant hepatocellular carcinoma: Potential targets by sulfasalazine. Cancer Sci 109: 2801-2810, 2018.

42. Bray F, Ferlay J, Soerjomataram I, Siegel RL, Torre LA and Jemal A: Global cancer statistics 2018: GLOBOCAN estimates of incidence and mortality worldwide for 36 cancers in 185 countries. CA Cancer J Clin 68: 394-424, 2018.

43. Molina JR, Yang P, Cassivi SD, Schild SE and Adjei AA: Non-small cell lung cancer: Epidemiology, risk factors, treatment and survivorship. Mayo Clin Proc 83: 584-594, 2008.

44. Bianco A, Perrotta F, Barra G, Malapelle U, Rocco D and De Palma R: Prognostic factors and biomarkers of responses to immune checkpoint inhibitors in lung cancer. Int J Mol Sci 20: 4931, 2019.

45. Ji X, Qian J, Rahman SM, Siska PJ, Zou Y, Harris BK, Hoeksema MD, Trenary IA, Heidi C, Eisenberg R, et al: xCT (SLC7A11)-mediated metabolic reprogramming promotes non-small cell lung cancer progression. Oncogene 37: 5007-5019, 2018.

46. Hu K, Li K, Lv J, Feng J, Chen J, Wu H, Cheng F, Jiang W, Wang J, Pei H, et al: Suppression of the SLC7A11/glutathione axis causes synthetic lethality in KRAS-mutant lung adenocarcinoma. J Clin Invest 130: 1752-1766, 2020.

47. Wang SJ, Li D, Ou Y, Jiang L, Chen Y, Zhao Y and Gu W: Acetylation is crucial for $\mathrm{p} 53$-mediated ferroptosis and tumor suppression. Cell Rep 17: 366-373, 2016.

48. Jennis M, Kung CP, Basu S, Budina-Kolomets A, Leu JIJ, Khaku S, Scott JP, Cai KQ, Campbell MR, Porter DK, et al: An African-specific polymorphism in the TP53 gene impairs p53 tumor suppressor function in a mouse model. Genes Dev 30 : 918-930, 2016

49. Okamoto K, Saito Y, Narumi K, Furugen A, Iseki K and Kobayashi M: Different mechanisms of cisplatin resistance development in human lung cancer cells. Biochem Biophys Res Commun 530: 745-750, 2020.

50. Otsubo K, Nosaki K, Imamura CK, Ogata H, Fujita A, Sakata S, Hirai F, Toyokawa G, Iwama E, Harada T, et al: Phase I study of salazosulfapyridine in combination with cisplatin and pemetrexed for advanced non-small-cell lung cancer. Cancer Sci 108: 1843-1849, 2017.
51. Winston JS, Ramanaryanan J and Levine E: HER-2/neu evaluation in breast cancer: Are we there yet? Am J Clin Pathol 121 (Suppl): S33-S49, 2004.

52. Chen MC, Hsu LL, Wang SF, Hsu CY, Lee HC and Tseng LM: ROS Mediate xCT-dependent cell death in human breast cancer cells under glucose deprivation. Cells 9: 1598, 2020.

53. Yang Y and Yee D: IGF-I regulates redox status in breast cancer cells by activating the amino acid transport molecule $\mathrm{xC}$-. Cancer Res 74: 2295-2305, 2014

54. Ruiu R, Rolih V, Bolli E, Barutello G, Riccardo F, Quaglino E, Merighi IF, Pericle F, Donofrio G, Cavallo F and Conti L: Fighting breast cancer stem cells through the immune-targeting of the $\mathrm{xCT}$ cysteine-glutamate antiporter. Cancer Immunol Immunother 68: 131-141, 2019.

55. Conti L, Bolli E, Di Lorenzo A, Franceschi V, Macchi F, Riccardo F, Ruiu R, Russo L, Quaglino E, Donofrio G and Cavallo F: Immunotargeting of the XCT cystine/glutamate antiporter potentiates the efficacy of HER2-targeted immunotherapies in breast cancer. Cancer Immunol Res 8: 1039-1053, 2020.

56. Ishiuchi S, Tsuzuki K, Yoshida Y, Yamada N, Hagimura N, Okado H, Miwa A, Kurihara H, Nakazato Y, Tamura M, et al: Blockage of $\mathrm{Ca}(2+)$-permeable AMPA receptors suppresses migration and induces apoptosis in human glioblastoma cells. Nat Med 8: 971-978, 2002.

57. Robert SM, Buckingham SC, Campbell SL, Robel S, Holt KT, Ogunrinu-Babarinde T, Warren PP, White DM, Reid MA, Eschbacher JM, et al: SLC7A11 expression is associated with seizures and predicts poor survival in patients with malignant glioma. Sci Transl Med 7: 289ra86, 2015.

58. Breemen MS, Wilms EB and Vecht CJ: Epilepsy in patients with brain tumours: Epidemiology, mechanisms, and management. Lancet Neurol 6: 421-430, 2007.

59. Sørensen MF, Heimisdóttir SB, Sørensen MD, Mellegaard CS, Wohlleben H, Kristensen BW and Beier CP: High expression of cysteine-glutamate antiporter $\mathrm{xCT}$ (SLC7A11) is an independent biomarker for epileptic seizures at diagnosis in glioma. J Neurooncol 138: 49-53, 2018.

60. Polewski MD, Reveron-Thornton RF, Cherryholmes GA, Marinov GK and Aboody KS: SLC7A11 overexpression in glioblastoma is associated with increased cancer stem cell-like properties. Stem Cells Dev 26: 1236-1246, 2017.

61. Tsuchihashi K, Okazaki S, Ohmura M, Ishikawa $M$, Sampetrean O, Onishi N, Wakimoto H, Yoshikawa M, Seishima R, Iwasaki Y, et al: The EGF receptor promotes the malignant potential of glioma by regulating amino acid transport system xc(-). Cancer Res 76: 2954-2963, 2016.

62. Long Y, Tao H, Karachi A, Grippin AJ, Jin L, Chang YE, Zhang W, Dyson KA, Hou AY, Na M, et al: Dysregulation of glutamate transport enhances treg function that promotes VEGF blockade resistance in glioblastoma. Cancer Res 80: 499-509, 2020.

63. Xu X, Zhang X, Wei C, Zheng D, Lu X, Yang Y, Luo A, Zhang K, Duan X and Wang Y: Targeting SLC7A11 specifically suppresses the progression of colorectal cancer stem cells via inducing ferroptosis. Eur J Pharm Sci 152: 105450, 2020.

64. Gout PW, Buckley AR, Simms CR and Bruchovsky N: Sulfasalazine, a potent suppressor of lymphoma growth by inhibition of the $\mathrm{x}(\mathrm{c})$-cystine transporter: A new action for an old drug. Leukemia 15: 1633-1640, 2001.

65. Wang W, Green M, Choi JE, Gijon M, Kennedy PD, Johnson JK, Liao P, Lang X, Kryczek I, Sell A, et al: CD8(+) T cells regulate tumour ferroptosis during cancer immunotherapy. Nature 569: 270-274, 2019.

66. Ye LF, Chaudhary KR, Zandkarimi F, Harken AD, Kinslow CJ, Upadhyayula PS, Dovas A, Higgins DM, Tan H, Zhang Y, et al: Radiation-induced lipid peroxidation triggers ferroptosis and synergizes with ferroptosis inducers. ACS Chem Biol 15: 469-484, 2020

This work is licensed under a Creative Commons Attribution-NonCommercial-NoDerivatives 4.0 International (CC BY-NC-ND 4.0) License. 